\title{
Thrombolysis with Systemic Recombinant Tissue Plasminogen Activator in Children: A Multicenter Retrospective Study
}

\author{
Çocuklarda Sistemik Rekombinant Doku Plazminojen Aktivatörü ile Tromboliz: Çok Merkezli \\ Bir Retrospektif Çalışma
}

\begin{abstract}
(D) Emine Zengin1, (D) Nazan Sarper1, (D) Arzu Yazal Erdem², (D) Işık Odaman Al33, (D) Melike Sezgin Evim4, (D) Neşe Yaralı2, (D) Burcu Belen5, (D) Arzu Akçay6, (D) Ayşen Türedi Yıldııı77, (D) Tuba Hilkay Karapınar³, (D) Adalet Meral Güneş4, (D) Sema Aylan Gelen¹, (D) Hale Ören8, (D) Lale Olcay5, (D) Birol Baytan4, (D) Hüseyin Gülen7, (D) Gülyüz Öztürk6, (D) Mehmet Fatih Orhan9 ${ }^{9}$, (D) Yeşim Oymak³, (D) Sibel Akpınar10, (D) Özlem Tüfekçi8, (D) Meryem Albayrak11, (D) Burçak Tatlı Güneş12, (D) Aylin Canpolat13, (D) Namık Özbek²

${ }_{1}^{1}$ Kocaeli University Faculty of Medicine, Department of Pediatrics, Division of Pediatric Hematology, Kocaeli, Turkey

2 University of Health Sciences Turkey, Ankara City Hospital, Department of Pediatrics, Division of Pediatric Hematology, Ankara, Turkey

3 University of Health Sciences Turkey, Dr. Behçet Uz Pediatrics and Pediatric Surgery Training and Research Hospital, Izmir, Turkey

4Uludağ University Faculty of Medicine, Department of Pediatrics, Division of Pediatric Hematology, Bursa, Turkey

${ }^{5}$ Başkent University Ankara Hospital, Clinic of Pediatrics, Division of Pediatric Hematology, Ankara, Turkey

${ }^{6}$ Acıbadem Mehmet Ali Aydınlar University Acıbadem Hospital, Clinic of Pediatric Hematology, Istanbul, Turkey

${ }^{7}$ Celal Bayar University Faculty of Medicine, Department of Pediatrics, Division of Pediatric Hematology, Manisa, Turkey

${ }^{8}$ Dokuz Eylül University Faculty of Medicine, Department of Pediatrics, Division of Pediatric Hematology, Izmir, Turkey

${ }^{9}$ Sakarya University Faculty of Medicine, Department of Pediatrics, Division of Pediatric Hematology, Sakarya, Turkey

10 University of Health Sciences Turkey, Kanuni Sultan Süleyman Training and Research Hospital, Clinic of Pediatrics, Division of Pediatric

Hematology, istanbul, Turkey

${ }_{11}$ Kırıkkale University Faculty of Medicine, Department of Pediatrics, Division of Pediatric Hematology, Kırıkkale, Turkey

12 University of Health Sciences Turkey, Tepecik Training and Research Hospital, Department of Pediatrics, Division of Pediatric Hematology, Izmir, Turkey

13istanbul Medeniyet University Göztepe Training and Research Hospital, Clinic of Pediatrics, Division of Pediatric Hematology, Istanbul, Turkey
\end{abstract}

\section{Abstract}

Objective: This study aimed to evaluate systemic thrombolysis experiences with recombinant tissue plasminogen activator (rtPA).

Materials and Methods: Retrospective data were collected from 13 Turkish pediatric hematology centers. The dose and duration of rtPA treatment, concomitant anticoagulant treatment, complete clot resolution (CCR), partial clot resolution (PCR), and bleeding complications were evaluated. Low-dose (LD) rtPA treatment was defined as $0.01-0.06 \mathrm{mg} / \mathrm{kg} / \mathrm{h}$ and high-dose (HD) rtPA as 0.1-0.5 $\mathrm{mg} / \mathrm{kg} / \mathrm{h}$.

Results: Between 2005 and 2019, 55 thrombotic episodes of 54 pediatric patients with a median age of 5 years (range: 1 day to 17.75 years) were evaluated. These patients had intracardiac thrombosis $(n=16)$, deep vein thrombosis (DVT) $(n=15)$, non-stroke arterial thrombosis $(n=14)$, pulmonary thromboembolism (PE) $(n=6)$, and stroke $(n=4)$. The duration from thrombus detection to rtPA initiation was a median of $12 \mathrm{~h}$ (range: 2-504 h) and it was significantly longer

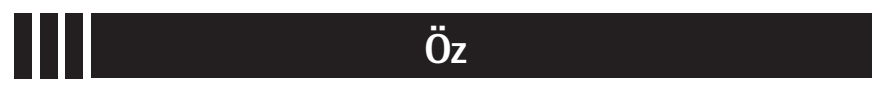

Amaç: Geriye dönük çok merkezli bir çalışma düzenleyerek, çocuk hematoloji uzmanlarının sistemik rekombinant doku plazminojen aktivatörü (rtPA) kullanımı ile ilgili deneyimlerinin ortaya konması amaçlandı

Gereç ve Yöntemler: Türkiye'deki 13 pediatrik hematoloji merkezinden geriye dönük veriler toplandı. rtPA tedavisinin dozu ve süresi, eşzamanlı antikoagülan tedavi, tam pıhtı erimesi (TPE), kısmi pıhtı erimesi (KPE) ve kanama komplikasyonları değerlendirildi. Düşük doz (DD) rtPA tedavisi 0,01-0,06 mg/kg/saat ve yüksek doz (YD) $0,1-0,5 \mathrm{mg} / \mathrm{kg} / \mathrm{saat}$ olarak tanımlandı.

Bulgular: 2005-2019 yılları arasında ortanca yaşı 5 yıl (1 gün-17,75 yıl) olan 54 hastanın 55 trombotik epizodu değerlendirildi. Hastaların tanıları; intrakardiyak tromboz $(n=16)$, derin ven trombozu (DVT) $(n=15)$, inme dışı arteriyel tromboz $(n=14)$, pulmoner tromboemboli $(P E)(n=6)$ ve inme $(n=4)$ idi. Trombüs saptanmasından rtPA başlangıcına kadar geçen süre medyan 12 saat (2 sa-504 sa) idi ve inme, inme olmayan

๑Copyright 2021 by Turkish Society of Hematology

Turkish Journal of Hematology, Published by Galenos Publishing House

口回是 Address for Correspondence/Yazışma Adresi: Emine Zengin, Prof., M.D., Kocaeli University Faculty of Medicine, Department of Pediatrics, Division of Pediatric Hematology, Kocaeli, Turkey

Phone : +905325740502

E-mail : eminezengin@hotmail.com ORCID: orcid.org/0000-0002-7362-6130
Received/Geliş tarihi: January 14, 2021 Accepted/Kabul tarihi: August 18, 2021 


\section{Abstract}

in cases of DVT and PE compared to stroke, non-stroke arterial thrombosis, and intracardiac thrombosis ( $p=0.024)$. In $63.6 \%$ of the episodes, heparin was initiated before rtPA treatment. LD and HD rtPA were administered in 22 and 33 of the episodes, respectively. Concomitant anticoagulation was used in $90 \%$ and $36 \%$ of the episodes with LD and HD rtPA, respectively $(p=0.0001)$. Median total duration of LD and HD rtPA infusions was $30 \mathrm{~h}$ (range: 2-120 h) and $18 \mathrm{~h}(2-120 \mathrm{~h})$, respectively $(\mathrm{p}=0.044)$. Non-fatal major and minor bleeding rates were $12.5 \%$ and $16.7 \%$ for LD and $3.2 \%$ and $25.8 \%$ for HD rtPA, respectively. At the end of the rtPA infusions, CCR and PCR were achieved in $32.7 \%$ and $49.0 \%$ of the episodes, respectively. The most successful site for thrombolysis was intracardiac thrombosis. HD versus LD rtPA administration was not correlated with CCR/PCR or bleeding ( $p>0.05)$.

Conclusion: Systemic thrombolytic therapy may save lives and organs effectively if it is used at the right indications and the right times in children with high-risk thrombosis by experienced hematologists with close monitoring of recanalization and bleeding.

Keywords: Recombinant tissue plasminogen activator, Thrombolysis, Childhood thrombosis
Öz

arteriyel tromboz ve intrakardiyak tromboza kıyasla DVT ve PE için anlamlı olarak daha uzundu $(p=0,024)$. Atakların \%63,6'sında rtPA tedavisinden önce heparin başlandı. Atakların 22'sine DD ve 33'üne YD rtPA uygulandı. DD ve YD rtPA ataklarının sırasıyla \%90'ında ve $\% 36$ 'sında eş zamanlı antikoagülasyon kullanıldı $(p=0,0001)$. DD ve YD rtPA infüzyonlarının medyan toplam süresi sırasıyla 30 sa (2 sa$120 \mathrm{sa})$ ve $18 \mathrm{sa}(2 \mathrm{sa}-120 \mathrm{sa})$ idi $(p=0,044)$. Ölümcül olmayan majör ve minör kanama oranları DD için sırasıyla $\% 12,5, \% 16,7$ ve YD rtPA için $\% 3,2, \% 25,8$ idi. rtPA infüzyonlarının sonunda, atakların sırasıyla \%32,7'sinde TPE ve \%49'unda KPE elde edildi. Tromboliz için en başarılı bölge intrakardiyak trombozdu. YD'ye karşı DD rtPA uygulaması, TPE/ KPE veya kanama ile korele değildi $(p>0,05)$.

Sonuç: Yüksek riskli trombozlu çocuklarda sistemik trombolitik tedavi, deneyimli hematologlar tarafından rekanalizasyon ve kanamanın yakın takibi altında doğru endikasyon ve doğru zamanda kullanıldığında etkin bir şekilde hayat ve organ kurtarabilir.

Anahtar Sözcükler: Rekombinant doku plazminojen aktivatörü, Tromboliz, Çocukluk trombozu

\section{Introduction}

The incidence of pediatric thrombosis is increasing due to advancements in neonatal and pediatric intensive care. Central venous catheters (CVCs), infections, surgery, immobility, trauma, congenital heart disease (CHD), vasculitis, underlying cancer, and thrombophilia are important risk factors for thrombosis $[1,2,3]$. Endovascular and surgical interventions for CHD save many lives, but the development of thrombosis is a frequent complication of these procedures [4]. Anticoagulation with unfractionated heparin (UFH) or low-molecular-weight heparin (LMWH) is the routine management of pediatric acute thrombosis. Heparins are used to prevent propagation, embolization, and recurrence of acute thrombosis [5]. Anticoagulation does not provide rapid recanalization of the occluded vessels and $49 \%$ of surviving infants and children suffering from arterial thrombosis have long-term sequelae including extremity or organ loss or epilepsy $[1,3]$. Postthrombotic syndrome (PTS) is the most common long-term complication of deep vein thrombosis (DVT) of the extremities. It is a chronic condition characterized by the development of venous insufficiency that manifests clinically with swelling, pain, cramping, stasis, dermatitis, and ulceration of the affected extremity [6]. The incidence of pediatric PTS ranges from 3\% to $70 \%$ in median 2-year follow-up [6,7]. In cases of pediatric arterial thrombosis and DVT, mortality is nearly $4-9 \%$ and $2 \%$, respectively, with cranial and intracardiac thrombosis causing the most mortality $[1,8]$. Since 1990, off-label use of recombinant tissue plasminogen activator (rtPA) in newborns and children has shown that systemic thrombolytic therapy is very effective if it is used at the right indications in selected patients under close monitoring $[9,10,11,12,13,14]$. Endovascular thrombolysis requires expert interventional radiologists and general anesthesia, and the small vessel diameters of children frequently limit such procedures; in addition, this intervention may damage the vascular endothelium and induce new thrombosis. However, the intravenous administration of thrombolytics is practical [5]. In this retrospective study, the experiences of pediatric hematology centers with systemic thrombolysis are presented. This study may help in the timely referral of pediatric patients to hematologists, intensive care physicians, or cardiologists for thrombolysis in cases of organ-, limb-, and life-threatening thrombi.

\section{Materials and Methods}

This retrospective study was approved by the institutional ethics committee and approval was obtained from the patients' legal guardians at admission to the hospital for the release of medical data for scientific purposes without including patient identity. A questionnaire form was designed and submitted to all Turkish pediatric hematology centers via electronic mail to collect the medical data of patients $0-18$ years old who received systemic rtPA for thrombolysis. rtPA administrations for CVC clearance were not included in the study. Major surgery or central nervous system bleeding within 10 days and inability to maintain a platelet count of at least $50,000 / \mu \mathrm{L}$ with transfusion support or fibrinogen of at least $100 \mathrm{mg} / \mathrm{dL}$ were exclusion criteria. Concomitant heparin administration was at the decision of the attending physician. UFH dose of 5-10 U/ $\mathrm{kg} / \mathrm{h}$ and LMWH 
(enoxaparin) dose of $0.5 \mathrm{mg} / \mathrm{kg}$ twice daily were the prophylactic doses. Patients' age, sex, underlying disease, location of the thrombus, period from thrombus detection to rtPA administration, previous anticoagulant treatments, initial and maximum rtPA doses, concomitant anticoagulant treatments, total duration of rtPA infusions, bleeding complications, thrombus resolution, amputations, organ loss, and outcomes after 3 months of anticoagulation were evaluated. Low-dose (LD) rtPA treatment was defined as $0.01-0.06 \mathrm{mg} / \mathrm{kg} / \mathrm{h}$ and high-dose (HD) as $0.1-0.5 \mathrm{mg} / \mathrm{kg} / \mathrm{h}[5,15]$. The centers reported that for older children and adolescents receiving LD continuous infusion, the maximum dose per hour was not defined and rtPA doses exceeding $2 \mathrm{mg} / \mathrm{h}$ were administered. Major bleeding was defined as any intracranial or retroperitoneal bleeding, or bleeding resulting in a drop in hemoglobin of $2 \mathrm{~g} / \mathrm{dL}$, or requiring transfusion or leading to death as defined by the International Society on Thrombosis and Haemostasis bleeding scale [16]. Minor bleeding was defined as oozing at the site of intravenous or other indwelling catheters or minor epistaxis, hematoma, hematuria, or melena not resulting in a drop in hemoglobin.

Diagnosis of thrombosis and monitoring for clot resolution performed by color Doppler ultrasound examination, echocardiography, and/or computed tomographic angiography was acceptable. Complete clot resolution (CCR) was defined as objective radiologic imaging confirming recanalization of the occluded vessel and/or arterial thrombosis clinical examination showing equal pulse pressure, capillary return, and warm extremities. Partial clot resolution (PCR) was defined as objective radiologic imaging confirming partial recanalization of the occluded vessels. No resolution (NR) was defined as objective radiologic imaging confirming no change or thrombus extension in the occluded vessel.

Centers reported performing laboratory monitoring by measuring prothrombin time, activated partial thromboplastin time, fibrinogen, anti-factor $\mathrm{Xa}$, fibrin degradation products, D-dimer, platelet counts, and hemoglobin levels every 6-12 h. Patients were strictly monitored clinically for bleeding (oozing from puncture sites, gastrointestinal system bleeding, and signs of cerebral bleeding), any new emboli, allergic and anaphylactoid reactions, laryngeal edema, orolingual angioedema, rash, and urticaria. Centers also monitored blood pressure, heart rate, and respiration rate. If minor bleeding developed, the rtPA dose was either deescalated or rtPA was withdrawn transiently. They stopped the treatment and infused fresh frozen plasma (FFP) and/or red blood cells if major bleeding developed. If recanalization was seen in imaging studies, rtPA was stopped.

\section{Statistical Analysis}

For calculation of median and percentage, descriptive analysis was used; for comparison of two independent variables, the Mann-Whitney $U$ test was used; and for comparison of more than two independent variables, Kruskal-Wallis $\mathrm{H}$ tests were used. Values of $p<0.05$ were defined as significant. All analyses were performed using SPSS version 13 (SPSS Inc., Chicago, IL, USA).

\section{Results}

Thirteen pediatric hematology centers participated in the study. Questionnaire forms for patients aged 0-18 years who received systemic rtPA (alteplase) between May 2005 and December 2019 were evaluated. The study included 55 thrombotic episodes of 54 patients ( 24 male, 30 female) with a median age of 5 years (range: 1 day to 17.75 years). Two episodes (intracardiac and diffuse pulmonary thromboembolism) of a patient with CHAPLE syndrome were included. Eight of these cases were already published $[11,17]$. Table 1 shows the characteristics of patients and treatment outcomes. Patients with intracardiac and nonstroke arterial thrombosis were younger than patients with pulmonary thromboembolism, DVT, and stroke $(p=0.001)$.

Out of 16 patients with intracardiac thrombus, five were preterm babies in the neonatal intensive care unit. They had umbilical catheters and ventilator support, and two of them also had septicemia. Five patients had CHD (one of them also had leukemia) and another two patients had cardiomyopathy. There were also patients with chronic pulmonary disease in the intensive care unit, acute lymphoblastic leukemia, lipin-1 deficiency (rhabdomyolysis + acute renal failure with hemodialysis), and CHAPLE syndrome (CD55 deficiency).

Out of 15 patients with DVT, five had hematological diseases (all with $\mathrm{CVCs}$ ) and three had metabolic diseases (propionic academia with CVC and Candida septicemia, hyperhomocysteinemia, and hyperlipidemia). There were also cases of Behçet's disease, pyelonephritis, exposure to trauma during football activity, Down syndrome, epileptic seizures, idiopathic Budd-Chiari syndrome, and a patient taking oral contraceptive pills.

In 14 patients with non-stroke arterial thrombosis, the underlying diseases were prematurity-associated in five patients (septicemia, respiratory distress syndrome, necrotizing enterocolitis, bronchopulmonary dysplasia) and one baby also had CHD. They also had umbilical catheters as a thrombus-provoking factor. CHD was also present in another four patients: Fallot tetralogy, ventricular septal defect (VSD) + patent foramen ovale + pulmonary hypertension, a newborn with aortic stenosis who underwent cardiac catheterization, and an infant with VSD who underwent transcatheter repair. Other patients had Kawasaki syndrome with coronary artery thrombus and pulmonary stenosis $(n=2)$, while there was also an infant with bronchiolitis in the pediatric intensive care unit and an adolescent in the postpartum period with inherited homozygous antithrombin (AT) deficiency. 


\begin{tabular}{|c|c|c|c|c|c|c|}
\hline & $\begin{array}{l}\text { Intracardiac thrombosis } \\
n=16\end{array}$ & $\begin{array}{l}\text { Deep venous } \\
\text { thrombosis } \\
n=15\end{array}$ & $\begin{array}{l}\text { Non-stroke } \\
\text { arterial } \\
\text { thrombosis } \\
n=14\end{array}$ & $\begin{array}{l}\text { Pulmonary } \\
\text { thromboembolism } \\
n=6\end{array}$ & $\begin{array}{l}\text { Stroke } \\
n=4\end{array}$ & $\mathbf{p}$ \\
\hline $\begin{array}{l}\text { Sex, } \\
\text { male/female }\end{array}$ & $7 / 9$ & $7 / 8$ & $5 / 9$ & $4 / 2$ & $2 / 2$ & 0.799 \\
\hline $\begin{array}{l}\text { Age, median } \\
\text { (range) }\end{array}$ & $\begin{array}{l}3 \text { years } \\
\text { (15 days- } 17.75 \text { years) }\end{array}$ & $\begin{array}{l}15 \text { years } \\
\text { ( } 1 \text { day- } 17 \text { years })\end{array}$ & $\begin{array}{l}0.6 \text { years } \\
\text { ( } 1 \text { day- } 17 \text { years })\end{array}$ & $\begin{array}{l}15 \text { years } \\
\text { (5-17 years) }\end{array}$ & $\begin{array}{l}11.5 \text { years } \\
(2.5-13.5 \text { years })\end{array}$ & 0.001 \\
\hline $\begin{array}{l}\text { Site of } \\
\text { thrombosis }\end{array}$ & $\begin{array}{l}7 \text { right atrium } \\
3 \text { right ventricle } \\
3 \text { left ventricle } \\
2 \text { left atrium } \\
1 \text { aortic and mitral valves }\end{array}$ & $\begin{array}{l}\text { Jugular, subclavian, } \\
\text { axillary, vena cava } \\
\text { inferior, vena porta, } \\
\text { iliac, renal }\end{array}$ & $\begin{array}{l}5 \text { lower extremity } \\
4 \text { upper extremity } \\
2 \text { coronary artery } \\
2 \text { renal artery } \\
1 \text { subclavian artery }\end{array}$ & Pulmonary & $\begin{array}{l}2 \text { venous sinus } \\
1 \mathrm{MCA} \\
1 \text { circle of Willis } \\
+ \text { left ophthalmic } \\
\text { artery }\end{array}$ & \\
\hline $\begin{array}{l}\text { Underlying } \\
\text { disease or } \\
\text { provoking } \\
\text { factors }\end{array}$ & $\begin{array}{l}5 \text { prematurityt } \\
4 \text { CHD } \\
2 \text { dilated CMP } \\
1 \text { CHD + leukemia } \\
1 \text { leukemia } \\
1 \text { lipin-1 deficiency } \\
1 \text { chronic pulmonary } \\
\text { diseaset } \\
1 \text { CHAPLE syndrome }\end{array}$ & $\begin{array}{l}3 \text { leukemia } \\
1 \text { AML-HSCT-pRTA-CS } \\
1 \text { Fanconi anemia-HSCT } \\
1 \text { hyperlipidemia } \\
1 \text { OC } \\
1 \text { Behçet's disease } \\
1 \text { pyelonephritist } \\
1 \\
\text { hyperhomocysteinemia } \\
1 \text { propionic acidemiat } \\
1 \text { epilepsy } \\
1 \text { trauma } \\
1 \text { Down syndrome + } \\
\text { obesity } \\
1 \text { idiopathic Budd- } \\
\text { Chiari syndrome }\end{array}$ & $\begin{array}{l}5 \text { prematurityt } \\
4 \text { CHD } \\
2 \text { Kawasaki } \\
\text { syndrome } \\
1 \text { inherited AT } \\
\text { deficiency } \\
1 \text { bronchiolitis in } \\
\text { PICUt } \\
1 \text { infant- } \\
\text { bacteriemiat }\end{array}$ & $\begin{array}{l}1 \text { Evans-CS } \\
1 \text { obesity- } \\
\text { postpartum } \\
1 \text { nephrotic } \\
\text { syndrome } \\
1 \text { Behçet's disease } \\
1 \text { MS-CS } \\
1 \text { CHAPLE } \\
\text { syndrome }\end{array}$ & $\begin{array}{l}1 \text { leukemia } \\
1 \text { aplastic anemia- } \\
\text { HSCT-progesterone } \\
1 \text { AOM- } \\
\text { mastoiditist } \\
1 \text { idiopathic }\end{array}$ & \\
\hline $\begin{array}{l}\text { Infection- } \\
\text { related } \\
\text { thrombosis, n }\end{array}$ & 2 & 2 & 5 & 0 & 1 & \\
\hline $\begin{array}{l}\text { Patients with } \\
\text { central venous } \\
\text { catheters, } \mathrm{n}\end{array}$ & 9 & 5 & 4 & 0 & 2 & \\
\hline $\begin{array}{l}\text { Patients with } \\
\text { malignancy, n }\end{array}$ & 2 & 4 & 0 & 0 & 1 & \\
\hline $\begin{array}{l}\text { Period from } \\
\text { thrombus } \\
\text { detection } \\
\text { to rtPA } \\
\text { administration } \\
\text { (h), median } \\
\text { (range) }\end{array}$ & $2(2-504)$ & $48(2-360)$ & $7(2-48)$ & $60(12-360)$ & $2(2-12)$ & 0.024 \\
\hline $\begin{array}{l}\mathrm{rtPA} \text { dose }(\mathrm{mg} / \\
\mathrm{kg} / \mathrm{h}), \text { median } \\
\text { (range) }\end{array}$ & $0.25(0.01-0.5)$ & $0.06(0.03-0.5)$ & $0.2(0.01-0.5)$ & $0.175(0.03-0.3)$ & $0.055(0.03-0.1)$ & 0.160 \\
\hline \multicolumn{7}{|c|}{ Patients on concomitant anticoagulation } \\
\hline $\begin{array}{l}\text { UFH, n (\%) } \\
\text { LMWH, n (\%) }\end{array}$ & $\begin{array}{l}1(6) \\
4(25)\end{array}$ & $\begin{array}{l}3(20) \\
7(46.6)\end{array}$ & $\begin{array}{l}3(21) \\
7(50)\end{array}$ & $\begin{array}{l}1(16.6) \\
2(33.3)\end{array}$ & $\begin{array}{l}2(50) \\
2(50)\end{array}$ & 0.046 \\
\hline $\begin{array}{l}\text { Duration of } \\
\text { rtPA infusion } \\
\text { (h), median } \\
\text { (range) }\end{array}$ & $15(2-36)$ & $18(6-120)$ & 30 (6-120) & $41(24-48)$ & $27(6-96)$ & 0.015 \\
\hline
\end{tabular}




\begin{tabular}{|c|c|c|c|c|c|c|}
\hline & $\begin{array}{l}\text { Intracardiac thrombosis } \\
n=16\end{array}$ & $\begin{array}{l}\text { Deep venous } \\
\text { thrombosis } \\
n=15\end{array}$ & $\begin{array}{l}\text { Non-stroke } \\
\text { arterial } \\
\text { thrombosis } \\
n=14\end{array}$ & $\begin{array}{l}\text { Pulmonary } \\
\text { thromboembolism } \\
n=6\end{array}$ & $\begin{array}{l}\text { Stroke } \\
n=4\end{array}$ & $p$ \\
\hline $\begin{array}{l}\text { Minor bleeding, } \\
\text { n (\%) }\end{array}$ & 3 (18.7) & $6(40)$ & 3 (21.4) & 0 & 0 & \\
\hline $\begin{array}{l}\text { Major bleeding, } \\
\text { n (\%) }\end{array}$ & 0 & 0 & $1(7.1)$ & $3(50)$ & 0 & 0.209 \\
\hline $\begin{array}{l}\text { Bleeding- } \\
\text { related death, } \\
\mathrm{n}(\%)\end{array}$ & 0 & 0 & 0 & 0 & 0 & \\
\hline \multicolumn{7}{|c|}{ Early outcome with rtPA } \\
\hline CCR, n (\%) & $10(62.5)$ & $2(13.3)$ & $3(21)$ & $3(50)$ & 0 & \\
\hline PCR, n (\%) & $6(37.5)$ & $9(60)$ & $6(42)$ & $3(50)$ & $3(75)$ & 0.006 \\
\hline $\begin{array}{l}\text { CCR after } 3 \\
\text { months of } \\
\text { anticoagulation }\end{array}$ & 5 (31.2) & 8 (53.3) & $5(35)$ & 2 (33.3) & $3(75)$ & 0255 \\
\hline $\begin{array}{l}\text { Total CCR after } \\
3 \text { months, } n \\
(\%)\end{array}$ & 15 (93) & $10^{*}(66.6)$ & $8^{* *}(57)$ & 5 (83.3) & $3(75)$ & 0.053 \\
\hline Sequelae/death & $\begin{array}{l}0 \text { sequelae } \\
1 \text { death (prematurity } \\
\text { septicemia) }\end{array}$ & $\begin{array}{l}1 \text { cirrhosis }^{* *} \\
2 \text { venous failure } \\
1 \text { death with VOD } \\
1 \text { lost to follow-up }\end{array}$ & $\begin{array}{l}\text { amputations } \\
-1 \text { leg } \\
-1 \text { arm } \\
-1 \text { finger } \\
1 \text { kidney atrophy } \\
2 \text { deaths related } \\
\text { to prematurity } \\
\text { complications }\end{array}$ & $\begin{array}{l}1 \text { pulmonary } \\
\text { hypertension }\end{array}$ & $\begin{array}{l}1 \text { unilateral } \\
\text { blindness }\end{array}$ & \\
\hline \multicolumn{7}{|c|}{$\begin{array}{l}\text { AML: Acute myeloblastic leukemia, AOM: acute otitis media, CCR: complete clot resolution, CHD: congenital heart disease, CMP: } \\
\text { cardiomyopathy, CS: corticosteroid, HSCT: hematopoietic stem cell transplantation, LMWH: low-molecular-weight heparin, MCA: middle } \\
\text { cerebral artery, MS: multiple sclerosis, OC: oral contraceptives, rtPA: recombinant tissue plasminogen activator, PCR: partial clot resolution, } \\
\text { PICU: pediatric intensive care unit, pRTA: proximal renal tubular acidosis, UFH: unfractionated heparin, VOD: veno-occlusive disease. }\end{array}$} \\
\hline
\end{tabular}

In cases of pulmonary thromboembolism, underlying diseases were nephrotic syndrome, immune dysregulation (Evans syndrome; the patient received corticosteroid), Behçet's disease, multiple sclerosis with corticosteroid treatment, CHAPLE syndrome (CD55 deficiency), and obesity and pregnancy in a 17-year-old patient.

Underlying diseases of the patients with stroke were hematological disease in two (leukemia; a female adolescent with aplastic anemia who underwent hematopoietic stem cell transplantation, having a CVC and receiving depot progesterone), infection in one (acute mastoiditis), and idiopathic thrombus in a toddler.

As provoking factors for thrombus, there were CVCs, infections, and malignancy in 20 (36.4\%), 10 (18.2\%), and 7 (12.7\%) of the thrombotic episodes, respectively. There was more than one thrombotic risk factor for some patients (Table 1).
In 63.6\% (35/55) of the episodes, heparin was initiated before rtPA treatment. LMWH was preferred in 33 episodes and UFH in only two episodes. Six patients received FFP to replace plasminogen before administration of rtPA; two of them were infants. The period from thrombus detection to rtPA initiation was a median of $12 \mathrm{~h}(2-504 \mathrm{~h})$; this period was significantly longer for DVT and pulmonary thromboembolism compared to stroke, non-stroke arterial, and intracardiac thrombi $(p=0.024)$. When all 55 episodes were evaluated, no correlation was found between this period and thrombus resolution $(p=0.75)$.

There was a wide range of administered rtPA doses and durations. In 14 episodes, dose escalation, in four episodes dose de-escalation, and in two episodes temporary withdrawal of rtPA due to bleeding was applied. The median initial and median maximum rtPA doses were both $0.1 \mathrm{mg} / \mathrm{kg} / \mathrm{h}(0.01-0.5)$. The total duration of rtPA infusions was a median of $18 \mathrm{~h}(2-120 \mathrm{~h})$ and 
it was significantly shorter for cardiac thrombosis compared to pulmonary and arterial thrombosis $(p=0.015)$.

In this study, 33 patients received HD rtPA. Only one center used a standard rtPA treatment protocol. They used HD rtPA for $6 \mathrm{~h}$ and repeated infusion after $24 \mathrm{~h}$ if thrombus resolution was not achieved. They also transfused FFP at $10-20 \mathrm{~mL} / \mathrm{kg}$ concomitantly. They did not use concomitant heparin. This center had five patients in this study. For four patients, they had to repeat rtPA infusions 3-6 times (totally for 18-36 h) and reported no bleeding complications. One of their patients with femoral and popliteal artery thrombus had CCR after only $6 \mathrm{~h}$ of rtPA infusion. Out of their five patients (two cardiac, one peripheral artery, one pulmonary thromboembolism, one
DVT), two achieved CCR. Eight more patients from other centers received HD rtPA for only 2-6 $\mathrm{h}$. When all patients receiving HD rtPA for $\leq 6 \mathrm{~h}$ (total duration) were evaluated, 2/9 achieved CCR with two minor bleeding complications. Patients receiving HD rtPA for $6 \mathrm{~h}$ at a time with or without repeated daily doses had two minor bleeding complications (2/13) and only two had CCR (2/13).

In Table 2, patients receiving HD rtPA infusion for longer than $6 \mathrm{~h} /$ day are presented. Patients 5, 6, and 16 received rtPA for 7-12 $\mathrm{h}$ daily whereas the others received rtPA without interruption. These 20 patients received HD rtPA for 12-120 $\mathrm{h}$ totally and ten achieved CCR; they had one major and six minor bleeding complications. In eight episodes, concomitant

\begin{tabular}{|c|c|c|c|c|c|}
\hline $\begin{array}{l}\text { Patient number } \\
\text { Age/sex }\end{array}$ & Underlying disease/site of thrombosis & $\begin{array}{l}\mathrm{rtPA} \text { dose, } \\
\mathrm{mg} / \mathrm{kg} / \mathrm{h} \\
\text { duration }\end{array}$ & $\begin{array}{l}\text { Concomitant } \\
\text { medicine }\end{array}$ & $\begin{array}{l}\text { Bleeding } \\
\text { complications } \\
\text { during rtPA } \\
\text { treatment }\end{array}$ & $\begin{array}{l}\text { Clot } \\
\text { resolution }\end{array}$ \\
\hline $\begin{array}{l}1 \\
14 \text { years/M }\end{array}$ & Trauma/right iliac and femoral veins & $\begin{array}{l}0.06 / 6 \mathrm{~h} \\
0.3 / 6 \mathrm{~h} \\
0.5 / 6 \mathrm{~h} \\
\text { Total } 18 \mathrm{~h}\end{array}$ & UFH first $6 \mathrm{~h}$ & No & No \\
\hline $\begin{array}{l}2 \\
3.5 \text { months/M }\end{array}$ & $\begin{array}{l}\text { Preterm, NEC, BPD, hydrocephaly after } \\
\text { intracranial bleeding, acute gastroenteritis, } \\
\text { microthrombus of left-hand fingers }\end{array}$ & $0.1 / 120 \mathrm{~h}$ & LMWH for 3 days & No & No \\
\hline $\begin{array}{l}3 \\
24 \text { months/F }\end{array}$ & Tetralogy of Fallot/right popliteal artery & $\begin{array}{l}0.3 / 4 \mathrm{~h} \\
0.4 / 12 \mathrm{~h} \\
\text { Total } 16 \mathrm{~h}\end{array}$ & - & Minor & $\mathrm{CR}$ \\
\hline $\begin{array}{l}4 \\
13.5 \text { years/F }\end{array}$ & $\begin{array}{l}\text { HSCT for aplastic anemia, CVC, depot } \\
\text { progesterone use/left internal carotid } \\
\text { artery, posterior communicant artery, left } \\
\text { ophthalmic artery }\end{array}$ & $\begin{array}{l}0.03 / 6 \mathrm{~h} \\
0.1 / 12 \mathrm{~h} \\
\text { Total } 18 \mathrm{~h}\end{array}$ & UFH & No & $\begin{array}{l}\text { PR/left } \\
\text { blindness }\end{array}$ \\
\hline $\begin{array}{l}5 \\
45 \text { months/M }\end{array}$ & $\begin{array}{l}\text { Propionic acidemia, Candida septicemia, } \\
\text { catheter/vena cava superior }\end{array}$ & $\begin{array}{l}0.5 / 6 \mathrm{~h} / \mathrm{day} \\
0.2 / 12 \mathrm{~h} / \mathrm{day}^{*} \\
\text { Total } 18 \mathrm{~h} \\
\end{array}$ & LMWH & Minor & $\mathrm{CR}$ \\
\hline $\begin{array}{l}6 \\
35 \text { months/M }\end{array}$ & JMML, catheter/left jugular vein & $\begin{array}{l}0.1 / 6 \mathrm{~h} / \text { day } \\
0.1 / 7 \mathrm{~h} / \text { day }^{*} \\
\text { Total } 13 \mathrm{~h}\end{array}$ & - & Minor & PR \\
\hline $\begin{array}{l}7 \\
14.5 \text { years/F }\end{array}$ & $\begin{array}{l}\text { Down syndrome/portal vein, Budd-Chari } \\
\text { syndrome }\end{array}$ & $0.2 / 18 \mathrm{~h}$ & FFP & No & PR \\
\hline $\begin{array}{l}8 \\
17 \text { years/F }\end{array}$ & $\begin{array}{l}\text { Obesity, postpartum/pulmonary arteries, } \\
\text { main femoral and popliteal veins }\end{array}$ & $0.2 / 24 \mathrm{~h}$ & - & No & PR \\
\hline $\begin{array}{l}9 \\
2.5 \text { months/F }\end{array}$ & $\begin{array}{l}\text { Preterm SGA, sepsis, RDS, CVC/left iliac and } \\
\text { femoral arteries }\end{array}$ & $0.3 / 24 \mathrm{~h}$ & FFP & No & No \\
\hline $\begin{array}{l}10 \\
5 \text { years/M }\end{array}$ & $\begin{array}{l}\text { Nephrotic syndrome/diffuse pulmonary } \\
\text { embolism }\end{array}$ & $\begin{array}{l}0.03 / 12 \mathrm{~h} \\
0.15 / 6 \mathrm{~h} \\
0.03 / 28 \mathrm{~h} \\
\text { Total } 46 \mathrm{~h}\end{array}$ & - & No & $\mathrm{CR}$ \\
\hline $\begin{array}{l}11 \\
8 \text { years/M }\end{array}$ & $\begin{array}{l}\text { Kawasaki syndrome/left coronary artery } \\
\text { aneurism }\end{array}$ & $\begin{array}{l}0.1 / 5 \mathrm{~h} \\
0.2 / 4 \mathrm{~h} \\
0.3 / 9 \mathrm{~h} \\
\text { Total } 18 \mathrm{~h}\end{array}$ & - & Minor & PR \\
\hline
\end{tabular}




\begin{tabular}{|c|c|c|c|c|c|}
\hline $\begin{array}{l}\text { Patient number } \\
\text { Age/sex }\end{array}$ & Underlying disease/site of thrombosis & $\begin{array}{l}\mathrm{rtPA} \text { dose, } \\
\mathrm{mg} / \mathrm{kg} / \mathrm{h} \\
\text { duration }\end{array}$ & $\begin{array}{l}\text { Concomitant } \\
\text { medicine }\end{array}$ & $\begin{array}{l}\text { Bleeding } \\
\text { complications } \\
\text { during rtPA } \\
\text { treatment }\end{array}$ & $\begin{array}{l}\text { Clot } \\
\text { resolution }\end{array}$ \\
\hline $\begin{array}{l}12 \\
1 \mathrm{day} / \mathrm{F}\end{array}$ & $\begin{array}{l}\text { Late prematurity/left subclavian artery (at } \\
\text { birth) }\end{array}$ & $0.4 / 48 \mathrm{~h}$ & UFH & Minor & No \\
\hline $\begin{array}{l}13 \\
6 \text { months/F }\end{array}$ & $\begin{array}{l}32 \text { weeks preterm, pneumonia, convulsion, } \\
\text { hemiparesis, CVC/left atrium }\end{array}$ & $0.1 / 12 \mathrm{~h}$ & - & No & CR \\
\hline $\begin{array}{l}14 \\
17.5 \text { years/M }\end{array}$ & $\begin{array}{l}\text { Dilated cardiomyopathy, progressive } \\
\text { muscular dystrophy/left ventricle }\end{array}$ & $\begin{array}{l}0.3 / 6 \mathrm{~h} \\
0.4 / 16 \mathrm{~h} \\
0.5 / 6 \mathrm{~h} \\
\text { Total } 28 \mathrm{~h}\end{array}$ & - & No & $\mathrm{CR}$ \\
\hline $\begin{array}{l}15 \\
11.7 \text { years/M }\end{array}$ & VSD/right ventricle & $0.4 / 12 \mathrm{~h}$ & - & Minor & CR \\
\hline $\begin{array}{l}16 \\
10.5 \text { years/F }\end{array}$ & $\begin{array}{l}\text { Chronic pulmonary disease, septicemia, } \\
\text { tracheostomy, CVC, mechanic ventilation/ } \\
\text { right atrium }\end{array}$ & $\begin{array}{l}0.1 / 6 \mathrm{~h} / \mathrm{day} \\
0.2 / 12 \mathrm{~h} / \mathrm{day}^{*} \\
\text { Total } 18 \mathrm{~h}\end{array}$ & LMWH & No & PR \\
\hline $\begin{array}{l}17 \\
12 \text { years/M }\end{array}$ & VSD + pulmonary stenosis & $\begin{array}{l}0.3 / 12 \mathrm{~h} \\
0.4 / 12 \mathrm{~h} \\
0.5 / 12 \mathrm{~h} \\
\text { Total } 36 \mathrm{~h}\end{array}$ & - & No & CR \\
\hline $\begin{array}{l}18 \\
15 \text { days/F }\end{array}$ & $\begin{array}{l}\text { Preterm, septicemia, umbilical catheter/ } \\
\text { right atrium }\end{array}$ & $\begin{array}{l}0.3 / 8 \mathrm{~h} \\
0.4 / 12 \mathrm{~h} \\
\text { Total } 20 \mathrm{~h}\end{array}$ & - & No & $\mathrm{CR}$ \\
\hline $\begin{array}{l}19 \\
3.5 \text { years } / F\end{array}$ & $\begin{array}{l}\text { Hypertension, right kidney atrophy/left } \\
\text { renal artery }\end{array}$ & $0.1 / 72 \mathrm{~h}$ & UFH & No & $\mathrm{CR}$ \\
\hline $\begin{array}{l}20 \\
12 \text { years/M }\end{array}$ & $\begin{array}{l}\text { Behçet's disease/right atrium, pulmonary } \\
\text { embolism vena cava inferior, bilateral iliac } \\
\text { veins, vena porta, vena hepatica }\end{array}$ & $\begin{array}{l}0.3 / 4 \mathrm{~h} \\
0.25 / 3 \mathrm{~h} \\
0.3 / 39 \mathrm{~h} \\
\text { Total } 46 \mathrm{~h}\end{array}$ & UFH & Major & CR \\
\hline \multicolumn{6}{|c|}{$\begin{array}{l}\text { BPD: Bronchopulmonary dysplasia, CVC: central venous catheter, F: female, JMML: juvenile myelomonocytic leukemia, LMWH: low-molecular-weight heparin, M: male, NEC: necrotizin } \\
\text { enterocolitis, UFH: unfractionated heparin, VSD: ventricular septal defect. } \\
\text { * These three patients received rtPA for 6-12 h daily whereas others received rtPA without interruption. }\end{array}$} \\
\hline
\end{tabular}

heparin was used. FFP was used in only two episodes. The numbers were small for statistical comparison of the infusion period concerning thrombus resolution and bleeding complications. In HD rtPA treatment, a longer infusion period compared to $\leq 6 \mathrm{~h}$ seemed more effective (CCR 10/20 versus 2/9), but with more bleeding complications (six minor and one major bleeding episode in 20 patients versus two minor bleeding episodes in 9 patients).

Concomitant heparin was administered in 58.2\% (32/55) of the thrombotic episodes (10 UFH and 22 LMWH), in 24 episodes at the therapeutic dose and in eight episodes at the prophylactic dose. Therapeutic-dose heparin was preferred in patients receiving LD rtPA $(p<0.05)$. In intracardiac thrombosis, concomitant anticoagulant administration was less frequent compared to stroke or DVT $(p=0.046)$. In 18 episodes of this study, CCR was achieved, and in 11 of those, concomitant heparin was not used. In 10 of these episodes, the thrombi were intracardiac.

Four major non-fatal bleedings (7.2\%) developed; three were in patients with pulmonary thromboembolism (pulmonary hemorrhage, melena + hematemesis, hematuria) and one in a patient with coronary artery thrombosis (hematuria). Underlying diseases in the patients developing major bleeding were Kawasaki syndrome, Behçet's disease, Evans syndrome, and multiple sclerosis; they all received concomitant heparin and three of them received LD rtPA for 18-48 $\mathrm{h}$. FFP and packed red cells or only FFP or fibrinogen concentrate were administered as supportive treatment in major bleeding episodes. Antifibrinolytics were not used for any patient. There were minor bleedings in 12 episodes (21.8\%) that could be easily controlled; these were epistaxis, gingival bleeding, venipuncture site bleeding, CVC 
exit site or incision site bleeding, subcutaneous hematoma, and bone marrow puncture site bleeding. When patients with and without rtPA-related bleedings were compared, no relationship was found with age, gender, initial rtPA dose, maximum rtPA dose, site of thrombosis, total rtPA duration, or concomitant use of anticoagulants. In 22 patients receiving LD treatment, there were three major and four minor bleeding complications. In patients receiving LD continuous treatment, rtPA doses exceeding $2 \mathrm{mg} / \mathrm{h}$ were used in older children and adolescents. When major bleedings in patients receiving LD were evaluated, a dose above $2 \mathrm{mg} / \mathrm{h}$ was administered for only one adolescent with pulmonary thromboembolism. This patient received concomitant $\mathrm{LMWH}$ and had hematuria and injection site bleeding. Two other patients on LD treatment and with major bleeding also received concomitant LMWH.

As shown in Table 1, after rtPA administration, CCR and PCR were achieved in 18 (32.7\%) and 27 (49.0\%) of the episodes, respectively. In intracardiac thrombosis, CCR was achieved in 62.5\% and PCR in 37.5\%; thrombolysis was significantly more successful compared to patients with non-stroke arterial thrombosis (CCR 21\% and PCR 42\%) and patients with DVT (CCR 13.3\% and PCR 60\%) ( $p=0.006)$. Out of 16 patients with intracardiac thrombosis, CCR was achieved in two patients even with late rtPA administrations (7 and 21 days after diagnosis). The total duration of rtPA infusion was significantly shorter in intracardiac thrombosis compared to pulmonary thromboembolism and non-stroke arterial thrombosis. Physicians discontinued treatment when CCR was achieved. Two patients achieving only PCR after systemic thrombolysis with rtPA underwent endovascular thrombectomy: a patient with Kawasaki syndrome having coronary artery thrombus and another patient with left iliac and femoral vein thrombosis. The latter patient also developed a thrombus in the contralateral extremity in the following week under LMWH treatment. rtPA was administered again but due to NR with the second thrombus, endovascular thrombectomy was performed. Off-label rivaroxaban was started with special approval from the health authority.

There were two patients with unilateral renal artery thrombosis. The patient with inherited AT deficiency presented with left renal artery thrombosis in the postpartum period and left kidney atrophy developed. A 3.5-year-old patient with preexistent right kidney atrophy presented with left renal artery thrombosis and achieved CCR. There was an 11-year-old patient with pyelonephritis associated with left renal vein thrombosis (RVT); rtPA treatment was started 7 days after the diagnosis and PCR was achieved. This patient also already had right kidney atrophy at presentation. Unilateral blindness developed in a patient after stroke; the etiology of the thrombus was otitis media and mastoiditis. Amputations were performed for three patients with arterial thrombosis of extremities or fingers after unsuccessful thrombolytic treatments. Cirrhosis developed in a patient with idiopathic Budd-Chiari syndrome; this patient underwent liver transplantation. Three patients died due to prematurity complications of septicemia + necrotizing enterocolitis + bronchopulmonary dysplasia and one transplanted patient with juvenile myelomonocytic leukemia died due to veno-occlusive disease. Anticoagulant treatments were continued with LMWH for a median of 3 months (1-6 months) in all surviving patients except three patients who received coumadin and rivaroxaban and one non-compliant patient. Acetylsalicylic acid was also used for seven patients with arterial, cardiac, and venous thrombosis. The patient with CHAPLE syndrome also received eculizumab. When the patients' outcomes were evaluated after 3 months of anticoagulation, CCR was increased to $74.5 \%$ from $32.7 \%$.

In Table 3, LD and HD rtPA treatments are compared in terms of characteristics of thrombotic episodes, bleeding complications, and treatment outcomes. Patients' median age and median duration from thrombus detection to rtPA administration were similar between patients receiving LD and HD treatment. Although the numbers were small for statistical power, there were more patients with intracardiac thrombosis in the HD group. In the LD group, the median duration of rtPA treatment was longer $(p=0.044)$ and concomitant anticoagulant administration was more frequent $(p=0.0001)$. In LD treatment 90\% (20/22) and in HD treatment $36 \%(12 / 33)$ of the patients received concomitant anticoagulants. The dose of rtPA was not correlated with clot resolution or bleeding ( $p>0.05)$.

\section{Discussion}

The lack of prospective trials for the treatment of pediatric thrombosis with rtPA is a serious dilemma for pediatricians. The Thrombolysis in Pediatric Stroke Study started in 2010 but was ended by the National Institutes of Health in 2013 due to lack of accrual [18]. In the pediatric antithrombotic therapy guidelines published in 2012, thrombolysis was recommended for the following indications [19]: a) limbthreatening or organ-threatening arterial thrombosis (via proximal extension) or femoral artery thrombosis that fails to respond to initial UFH therapy; b) bilateral RVT with evidence of renal impairment; c) symptomatic peripheral arterial catheterrelated thromboembolism; d) right atrial thrombosis related to CVC, especially $>2 \mathrm{~cm}$ and mobile; and e) thrombotic giant coronary artery aneurism in cases of Kawasaki syndrome. The authors were more cautious in thrombolysis of childhood acute ischemic stroke outside of research protocols [19]. Some authors also included the following conditions among strong indications for thrombolysis: superior vena cava syndrome; pulmonary embolism with hypotension or shock or resulting in right heart strain or myocardial necrosis; extensive venous thrombosis with total occlusion of venous flow, increased 


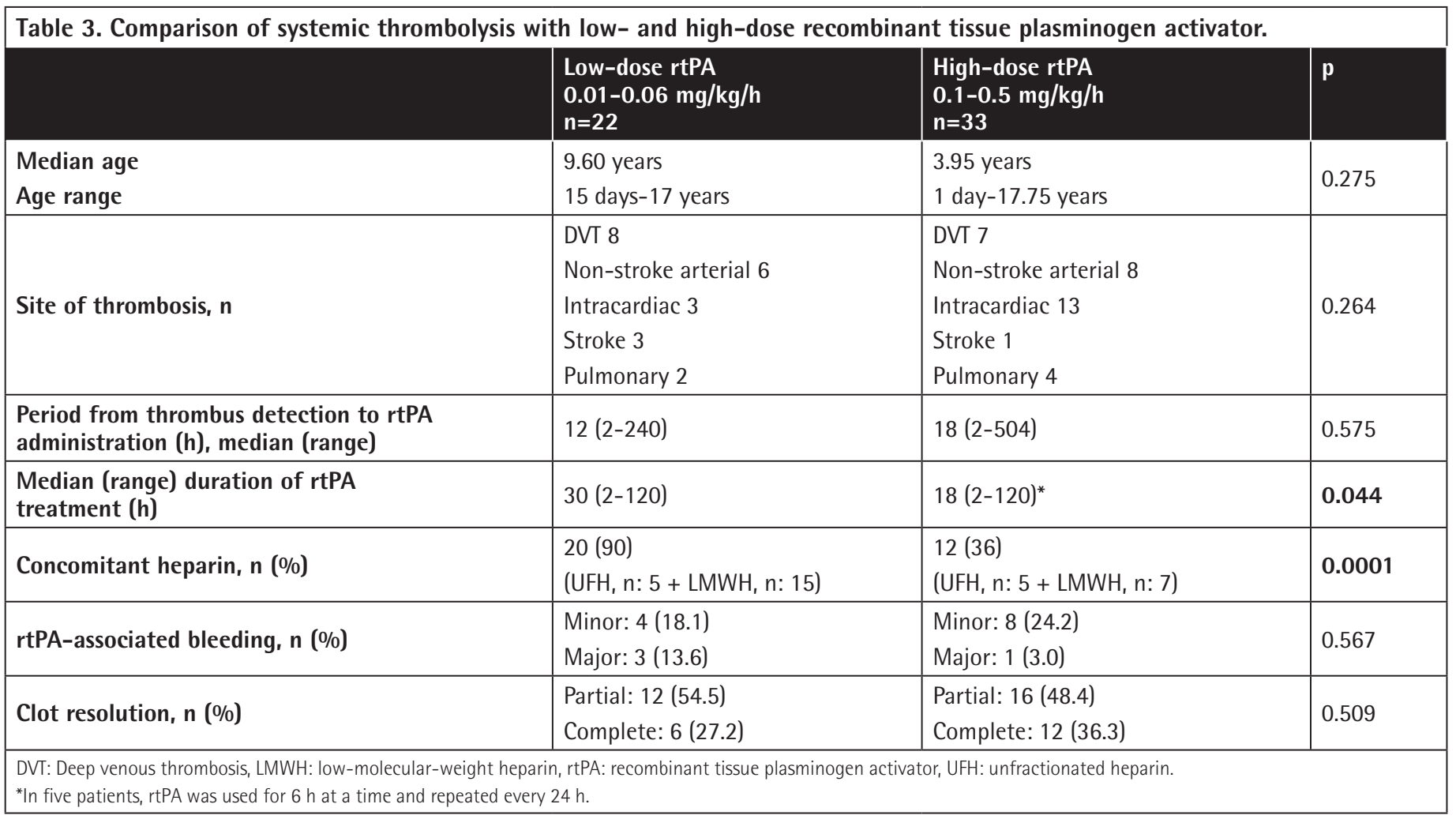

compartment pressures, and compromise of arterial blood flow; and CHD with shunt thrombosis and cerebral sinovenous thrombosis with neurological impairment and no improvement with anticoagulation or progressive thrombosis [5].

Tarango and Manco-Johnson [5] recommended systemic thrombolysis with LD treatment for 6-72 $\mathrm{h}$ and HD treatment for 2-6 $\mathrm{h}$ at a time, repeating the same doses if indicated over a period of $72 \mathrm{~h}$. In the current retrospective study, only one center applied HD treatment following this protocol, administering rtPA for $6 \mathrm{~h}$ at a time and repeating the infusion every $24 \mathrm{~h}$ if there was no clot resolution. However, they repeated the infusions for 6 days for some patients, longer than recommended by Tarango and Manco-Johnson [5]. Other centers used continuous infusions of LD and HD rtPA for a maximum of $120 \mathrm{~h}$ or stopped infusions earlier when CCR was achieved or bleeding complications occurred. When HD rtPA infusions for longer than $6 \mathrm{~h} /$ day were compared to HD for 2-6 h/day, bleeding complications were more frequent in infusions for $>6$ $\mathrm{h}$ (six minor bleedings and one major bleeding in 20 thrombotic episodes versus two minor bleedings in 13 thrombotic episodes). However, with LD continuous infusion (2-120 h), there were also four minor and three major bleeding episodes in 22 thrombotic episodes. In this series, major bleeding was more frequent with LD rtPA compared to HD rtPA. Centers administered continuous LD without any dose limitation per hour, exceeding $2 \mathrm{mg} / \mathrm{h}$ in older children and adolescents. Although bleeding episodes were non-fatal in this retrospective study, to be on the safe side, administration of continuous LD rtPA for longer than $72 \mathrm{~h}$ and/or exceeding $2 \mathrm{mg} / \mathrm{h}$ cannot be recommended, similar to administration of HD rtPA for longer than $6 \mathrm{~h}$ at a time.

Similar to our study, LD rtPA was shown to be effective and safe in a group of patients including preterm neonates and children [15]. In the present study, when LD and HD rtPA treatments were compared, there was no difference in clot resolution, but with $L D$ rtPA, the median duration of treatment was longer and physicians preferred concomitant heparin administration more frequently. This may be explained by physicians' fear of bleeding complications in HD treatment. CCR was achieved in $32.7 \%$ and PCR in $49.0 \%$ of the thrombotic episodes at the end of rtPA infusions. Similar to our results, in a study of 46 children receiving systemic rtPA, Ansah et al. [12] found that CCR and PCR/NR were not related to initial and maximum rtPA doses, duration of rtPA, or mean time from diagnosis to treatment. They reported that CCR and PCR were achieved in 46\% and 22\%, respectively, with a slightly higher CCR rate than that seen in our study. They also reported that bleeding complications occurred in 33\% of the patients and bleeding-related death in 4.3\%, and bleeding complications were related to median initial rtPA dose (0.10 mg/kg/h vs. $0.03 \mathrm{mg} / \mathrm{kg} / \mathrm{h}$ ) [12]. In the present study, in $63.6 \%$ of the episodes, heparin was initiated before rtPA treatment and concomitant heparin was administered to $90 \%$ of the patients receiving LD rtPA. Concomitant heparin was used to prevent proximal clot extension during rtPA infusion. 
The median duration of rtPA treatment was $30 \mathrm{~h}$ (range: 2-120 h) with LD treatment and $18 \mathrm{~h}(2-504 \mathrm{~h})$ with HD. In Wang et al.'s [15] study, the duration of treatment was 4-48 $\mathrm{h}$ with LD and 1-24 h with HD; complete lysis was achieved in 97\% of 29 patients with arterial thrombus, intracardiac thrombus, and DVT presented within 2 weeks. It is not easy to compare these studies and draw any conclusions due to the heterogeneity of the patients.

In the present series, early CCR and PCR rates were between $0 \%$ and $62.5 \%$ and between $37.5 \%$ and $75.0 \%$, respectively, for different thrombus locations, but thrombolysis was significantly more successful for intracardiac thrombi and pulmonary thromboembolism $(62.5 \%$ and $50 \% \quad \mathrm{CCR}$, respectively). Fortunately, after two endovascular thrombectomies following unsuccessful systemic rtPA administrations, and with 3 months of anticoagulation, CCR ranged between 57\% and 93\% for different thrombus locations.

In renal artery thrombus, rtPA treatment resulted in CCR in one of two patients. rtPA was also recommended for bilateral RVT with evidence of renal impairment [19]. In a study of five newborns with unilateral RVT, clot resolution was not successful with rtPA plus heparin [20]. However, in another newborn, CCR was achieved when rtPA was used even 6 days after the onset of thrombus [21].

In three of four patients with stroke, PCR was achieved with thrombolysis. In the patient with left median cerebral artery thrombosis, PCR was achieved with rtPA administration even $12 \mathrm{~h}$ after diagnosis. Low rates of bleeding complications were reported with rtPA in cases of pediatric ischemic stroke $[2,14]$, and reports about successful thrombolysis in pediatric stroke with systemic rtPA administered within the first $4 \mathrm{~h}$ are increasing $[2,13,22,23]$.

For a 12-year-old patient with Behçet's disease, rtPA was life-saving. Symptoms of thrombosis in multiple sites had developed within 15 days and systemic vasculitis was diagnosed. This patient was classified among the patients with pulmonary thromboembolism in this study, but he also had thrombi in the right atrium, vena cava inferior, bilateral iliac veins, vena porta, and vena hepatica. Administration of rtPA at $0.3 \mathrm{mg} / \mathrm{kg} / \mathrm{h}$ for $46 \mathrm{~h}$ and concomitant UFH led to CCR. In this patient, there was major bleeding, which was controlled with temporary drug withdrawal and transfusion. There were four major non-fatal bleeding episodes (7.2\%) in 55 systemic rtPA administrations. In addition to blood components, tranexamic acid may be used for bleeding complications. The use of antifibrinolytic agents for treating symptomatic intracranial hemorrhage that develops as a complication of thrombolytic therapy was also suggested [24]. Antifibrinolytics are available for urgent use compared to blood components.
In this series, CCR was achieved in only $13.3 \%$ of DVT episodes. In a pediatric study of 26 cases, there was no CCR in DVT, whereas CCR was achieved for $81 \%$ of arterial thrombi [10]. Successful thrombolysis in DVT with rtPA was reported in some pediatric studies $[12,25]$. In massive iliofemoral DVT with total occlusion of venous flow and/or compromise of arterial flow, there is a strong indication for systemic/catheter-directed (endovascular) thrombolysis or percutaneous mechanical thrombectomy to achieve venous patency [5]. Invasive interventions for thrombolysis of iliofemoral thrombosis are also technically possible in children aged 1-18 years and these approaches may reduce the risk or severity of PTS compared to standard anticoagulation alone [26]. Among the patients of the present study, endovascular thrombectomy was performed for one patient with iliofemoral thrombosis after unsuccessful systemic thrombolysis and extension of the thrombus to the contralateral extremity. However, thrombolysis with systemic rtPA was evaluated in this study.

In vitro studies show that rtPA has more rapid clot lysis activity and high affinity for fibrin, and it induces the binding of plasminogen to fibrin; due to these characteristics, it is recommended in newborns and children over other thrombolytics $[19,27]$. In the present study, alteplase was used with a half-life of 3-5 min; a short half-life provides bleeding control by transient drug withdrawal or decreasing the dose. UFH should be preferred for concomitant anticoagulation due to its short half-life. As a general practice, thrombolysis is attempted for vessel occlusions of up to 14 days due to the decreased response in cases of older thrombi [5]. Similar to our study, Ansah et al. [12] found no correlation between clot resolution and period from diagnosis to rtPA administration. In their study, the mean time from diagnosis to treatment was $36.0 \pm 16.8 \mathrm{~h}$ versus $18.1 \pm 5.3 \mathrm{~h}$ for CCR and PCR/NR, respectively.

\section{Study Limitations}

There are limitations of this study due to its retrospective multicenter nature and the heterogeneity of the patient population. For some patients, thrombolysis was initiated 2 weeks after thrombus presentation. Participating centers generally had no standard protocols for thrombolysis. Only some centers used concomitant heparin, either in therapeutic or prophylactic doses. Imaging studies for diagnosis and monitoring were not standardized. Data on serum fibrinogen, fibrin degradation products, anti-factor $\mathrm{X} \mathrm{a}$, prothrombin time, and activated partial thromboplastin time were not presented in this study. Thrombosis requiring thrombolysis is rare in children compared to adults and each center was already trying to develop its own experiences with thrombolysis during the 14-year study period. Prospective multicenter pediatric studies must be planned using standard protocols with the administration of continuous LD rtPA for 6-72 $\mathrm{h}$ and HD for not more than $6 \mathrm{~h}$ at a time. 


\section{Conclusion}

Systemic thrombolytic therapy with LD or HD rtPA may save lives and organs effectively if it is used for the right indications and at the right times in children with high-risk thrombosis. This therapy should be applied by experienced hematologists under close monitoring of recanalization and bleeding.

\section{Acknowledgments}

Thanks to all contributing centers and patients' guardians for sharing patient data. Thanks also to the steering committee of the Turkish Pediatric Hematology Association for their help in achieving communication and collaboration among the participating pediatric hematology centers.

\section{Ethics}

Ethics Committee Approval: This retrospective study was approved by the institutional ethics committee.

Informed Consent: Approval was obtained from the patients' legal guardians at admission to the hospital for the release of medical data for scientific purposes without including patient identity.

\section{Authorship Contributions}

Surgical and Medical Practices: E.Z., N.S., A.Y.E., I.O.A., M.S.E., N.Y., B.B., A.A., A.T.Y., T.H.K., A.M.G., S.A.G., H.Ö., L.O., B.B., H.G., G.Ö., M.F.O., Y.O., S.A., Ö.T., M.A., B.T.G., A.C., N.Ö.; Concept: E.Z.; Data Collection or Processing: E.Z., A.Y.E., I.O.A., M.S.E., N.Y., B.B., A.A., A.T.Y., T.H.K., A.M.G., H.Ö., L.O., B.B., H.G., G.Ö., M.F.O., Y.O., S.A., Ö.T., M.A., B.T.G., A.C., N.Ö.; Analysis or Interpretation: E.Z.; Literature Search: E.Z., N.S.; Writing: E.Z., N.S.

Conflict of Interest: No conflict of interest was declared by the authors.

Financial Disclosure: The authors declared that this study received no financial support.

\section{References}

1. Monagle $P$, Newall $F$, Barnes $C$, Savoia $H$, Campbell J, Wallace $T$, Crock C. Arterial thromboembolic disease: a single-centre case series study. J Paediatr Child Health 2008;44:28-32.

2. Nasr DM, Biller J, Rabinstein AA. Use and in-hospital outcomes of recombinant tissue plasminogen activator in pediatric arterial ischemic stroke patients. Pediatr Neurol 2014;51:624-631.

3. Oren H, Devecioğlu Ö, Ertem M, Vergin C, Kavaklı K, Meral A, Canatan D, Toksoy H, Yıldız İ, Kürekçi E, Özgen Ü, Öniz H, Gürgey A. Analysis of pediatric thrombotic patients in Turkey. Pediatr Hematol Oncol 2004;21:573-583.

4. Olgun H, Buyukavci M, Ceviz N, Sahin IO, Yildirim ZK, Colak A, Tekgunduz $\mathrm{KS}$, Caner I. Clinical experience with recombinant tissue plasminogen activator in the management of intracardiac and arterial thrombosis in children. Blood Coagul Fibrinolysis 2014;25:726-730.

5. Tarango C, Manco-Johnson MJ. Pediatric thrombolysis: a practical approach. Front Pediatr 2017;5:260.
6. Kuhle S, Koloshuk B, Marzinotto V, Bauman M, Massicotte P, Andrew M, Chan A, Abdolell M, Mitchell L. A cross-sectional study evaluating postthrombotic syndrome in children. Thromb Res 2003;111:227-233.

7. Goldenberg NA, Donadini MP, Kahn SR, Crowther M, Kenet G, Nowak-Göttl U, Manco-Johnson MJ. Post-thrombotic syndrome in children: a systematic review of frequency of occurrence, validity of outcome measures, and prognostic factors. Haematologica 2010;95:1952-1959.

8. Monagle $\mathrm{P}$, Adams M, Mahoney M, Ali K, Barnard D, Bernstein M, Brisson L, David M, Desai S, Scully MF, Halton J, Israels S, Jardine L, Leaker M, McCusker P, Silva M, Wu J, Anderson R, Andrew M, Massicotte MP. Outcome of pediatric thromboembolic disease: a report from the Canadian Childhood Thrombophilia Registry. Pediatr Res 2000;47:763-766.

9. Kennedy LA, Drummond WH, Knight ME, Millsaps MM, Williams JL. Successful treatment of neonatal aortic thrombosis with tissue plasminogen activator. J Pediatr 1990;116:798-801.

10. Newall F, Browne M, Savoia H, Campbell J, Barnes C, Monagle P. Assessing the outcome of systemic tissue plasminogen activator for the management of venous and arterial thrombosis in pediatrics. J Pediatr Hematol Oncol 2007;29:269-273.

11. Evim MS, Bostan Ö, Baytan B, Semizel E, Günes AM. Thrombolysis with recombinant tissue plasminogen activator in 7 children. Clin Appl Thromb Hemost 2013;19:574-577.

12. Ansah DA, Patel KN, Montegna L, Nicholson GT, Ehrlich AC, Petit CJ. Tissue plasminogen activator use in children: bleeding complications and thrombus resolution. J Pediatr 2016;171:67-72.e1-2.

13. Tabone L, Mediamolle N, Bellesme C, Lesage F, Grevent D, Ozanne A, Naggara O, Husson B, Desguerre I, Lamy C, Denier C, Kossorotoff M. Regional pediatric acute stroke protocol: initial experience during 3 years and 13 recanalization treatments in children. Stroke 2017;48:2278-2281.

14. Amlie-Lefond C, Shaw DWW, Cooper A, Wainwright MS, Kirton A, Felling RJ, Abraham MG, Mackay MT, Dowling MM, Torres M, Rivkin MJ, Grabowski EF, Lee S, Kurz JE, McMillan HJ, Barry D, Lee-Eng J, Ichord RN. Risk of intracranial hemorrhage following intravenous tPA (tissue-type plasminogen activator) for acute stroke is low in children. Stroke 2020;51:542-548.

15. Wang $M$, Hays $T$, Balasa $V$, Bagatell $R$, Gruppo R, Grabowski EF, Valentino LA, Tsao-Wu G, Manco-Johnson MJ; Pediatric Coagulation Consortium. Low dose tissue plasminogen activator thrombolysis in children. J Pediatr Hematol Oncol 2003;25:379-386.

16. Levine MN, Raskob G, Landefeld S, Kearon C. Hemorrhagic complications of anticoagulant treatment. Sixth ACCP Consensus Conference on Antithrombotic Therapy. Chest 2001;119:108-121.

17. Babayigit A, Cebeci B, Buyukkale G, Semerci SY, Bornaun H, Oztarhan K, Gokce M, Cetinkaya M. Treatment of neonatal fungal infective endocarditis with recombinant tissue plasminogen: activator in a low birth weight infant case report and review of the literature. Mycoses 2015;58:578-581.

18. Rivkin MJ, deVeber G, Ichord RN, Kirton A, Chan AK, Hovinga CA, Gill JC, Szabo A, Hill MD, Scholz K, Amlie-Lefond C. Thrombolysis in Pediatric Stroke Study. Stroke 2015;46:880-885.

19. Monagle P, Chan AKC, Goldenberg NA, Ichord RN, Journeycake JM, NowakGöttl U, Vesely SK. Antithrombotic therapy in neonates and children: Antithrombotic Therapy and Prevention of Thrombosis, 9th ed: American College of Chest Physicians Evidence-Based Clinical Practice Guidelines. Chest 2012;141(2 Suppl):e737S-e801S.

20. Niada F, Tabin R, Kayemba-Kay's S. Spontaneous neonatal renal vein thromboses: Should we treat them all? A report of five cases and a literature review. Pediatr Neonatol 2018:59:281-287.

21. Wang KY, Tsai LW, Chen CM. Tissue plasminogen activator therapy for renal venous thrombosis. Arch Dis Child Fetal Neonatal Ed 2008;93:F397-398.

22. Sampaio I, Abecasis F, Quintas S, Moreno T, Camilo C, Vieira M, Pinho e Melo T, Correia M. Successful intravenous thrombolysis in a 14-year-old boy with ischemic stroke. Pediatr Emerg Care 2011;27:541-543. 
23. Viaro $F$, Manara $R$, Farina $F$, Palmieri $A$, Rocca $F D$, Ballotta $E$, Baracchini C. Successful systemic thrombolysis in an adolescent with acute ischemic stroke. Neurologist 2015;20:48-50.

24. French $\mathrm{KF}$, White J, Hoesch RE. Treatment of intracerebral hemorrhage with tranexamic acid after thrombolysis with tissue plasminogen activator. Neurocrit Care 2012;17:107-111.

25. Tan $H$, Kizilkaya $M$, Alper $F$, Becit N, Kürşat $H$. Thrombolytic therapy with tissue plasminogen activator for superior vena cava thrombosis in an infant with sepsis. Acta Paediatr 2005;94:239-241.
26. Gaballah M, Shi J, Kukreja K, Raffini L, Tarango C, Keller M, Krishnamurthy G, Racadio J, Patel M, Cahill AM. Endovascular thrombolysis in the management of iliofemoral thrombosis in children: a multi-institutional experience. J Vasc Interv Radiol 2016;27:524-530.

27. Ouriel $K$, Kolassa M, DeWeese JA, Green RM. Economic implications of thrombolysis or operation as the initial treatment modality in acute peripheral arterial occlusion. Surgery 1995;118:810-814. 